\title{
TRALOC \\ Teleoperated Snake-Robot with Tracks on each Segment, for Searching Victims in Collapsed Buildings
}

\section{Conference Paper}

\section{Author(s):}

Bleiker, Tobias; Darbre, Jérôme; Droll, Santiago; Heer, Flavio; Hunziker, Dominique; Winandy, Tom; Fischer, Wolfgang; Siegwart, Roland

\section{Publication date:}

2012

\section{Permanent link:}

https://doi.org/10.3929/ethz-a-010025786

\section{Rights / license:}

In Copyright - Non-Commercial Use Permitted 


\title{
TRALOC - TELEOPERATED SNAKE-ROBOT WITH TRACKS ON EACH SEGMENT, FOR SEARCHING VICTIMS IN COLLAPSED BUILDINGS
}

\author{
T. BLEIKER, J. DARBRE, S. DROLL, F. HEER, D. HUNZIKER, T. WINANDY \\ ETH Zürich, 8092 Zürich, Switzerland \\ W. FISCHER, R. SIEGWART \\ Autonomous System Lab, ETH Zürich, \\ Tannenstrasse 3, 8092 Zürich, Switzerland
}

\begin{abstract}
This paper presents the "Traloc", a mobile remote-controlled snakelike robot, which is designed to search victims in the debris after earthquakes. It consists of 5 similar elements with motorized tracks on each side; 4 joints with 2 DOF each in between, a "head-unit" with camera and the cable which connects it to the control unit. Thanks to the actively driven tracks in each element, the mobility of the snake is significantly higher than comparable systems, where only the joints are actuated. With this advanced mobility, our robot is already able to overcome steps and gaps which are approximately the size of one segment - with only 5 elements in total. By implementing an innovative actuation principle for the joints which is based on a direct chain-drive, the entire robot could be realized very lightweight and without the need of pneumatic air-supply - which is crucial for reliability and future upgrades towards a wireless version running on batteries.
\end{abstract}

Keywords: Field Robot, Mechanism Design, Search and Rescue, Hybrid Track Snake robot,

\section{Introduction}

Our goal is to provide a system which is capable to move through the debris resulting after an earthquake and to support the first responders in localizing buried victims. The situation on site is very difficult due to the expectations of the local population, the problematic logistics and the environmental influences. This is in an increase of pressure and delay in the rescue. Because of the dramatically decreasing chance of survival after 72 hours, it is indispensable that the rescue is as much facilitated as possible. 
Our response to this situation is a robot called Traloc, which supplements a more precise and faster possibility to locate victims to the already existing methods (dogs, borescopes, etc.). The target is to develop and construct such a robot which is able move through the debris after an earthquake and handle the specifications that are a result of the challenging terrain.

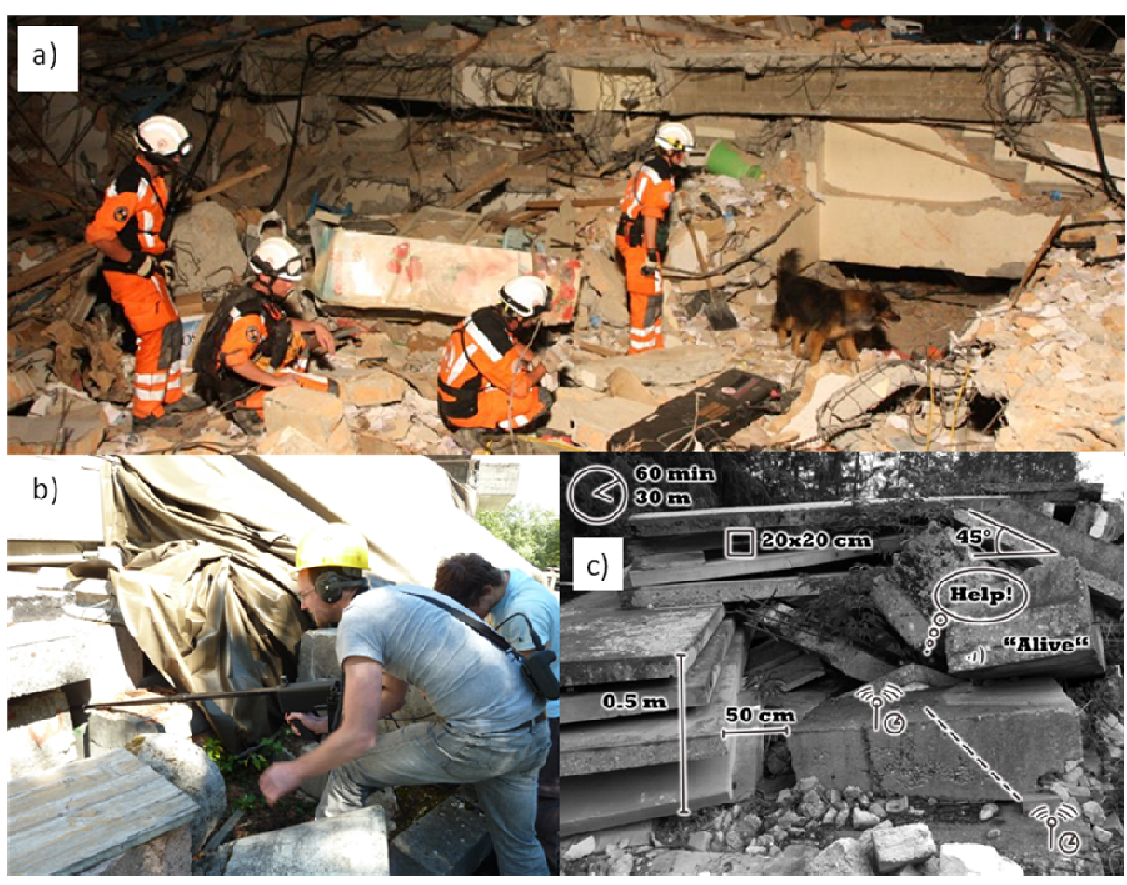

Figure 1. Typical environment in a collapsed building

(a) Photo with a classical rescue-team at work, (b) current search instrument, (c) Detail-view of typical obstacles, with the most difficult challenges for robot locomotion highlighted.

After analyzing the requirements in more detail in collaboration with our partners from the Swiss Military Disaster Relief, the following specifications for such a robot were defined (see Figure 1-c):

- Need to localize a victim precisely

- The system should be easy to control from the outside of the debris

- Should be transportable as standard-luggage $(<30 \mathrm{~kg})$

- A gap of up to half a meter must be overcome

- A step of up to half a meter must be overcome

- Holes with a minimal size of 20x20 cm must be passed

- The robot should be able to pass a slope of up to $45^{\circ}$

- A minimum action time of about an hour is required

- The path which the robot needs to travel can be up to $30 \mathrm{~m}$ 


\section{State of the art}

Currently the standard method for localizing buried victims is searching with dogs. However, the animals tend to be too large to directly reach the buried victims. For this reason technical support aids, such as telescopic cameras and acoustic sensors, are normally used to verify the approximate position of the victims indicated by the dog. Unfortunately all currently available technical equipment in this field is insufficient. Different robotic approaches are being investigated to address the existing problems.

One possible solution is the development of airborne robots [1] with the main advantage of overcoming any type of obstacle regarding the terrain. However the payload is limited and the spatial requirements and energy consumption are too high.

Robots with wheels [2] or tracks [3] and active joints also have a very good ability to overcome large obstacles, but still tend to be too large for accessing the most narrow gaps in the debris $(20 \times 20 \mathrm{~cm}$ are specified).

Snakelike robots inspired by nature [4], which are more flexible and potentially smaller than common vehicles, are very difficult to control due to the high number of required elements and the complex gait patterns of a snake.

A combination of a snakelike system with wheels [5] or tracks [6, 7] allows achieving similar mobility with a smaller number of elements and significantly simplified control. While a system with wheels is easier to realize regarding the mechanical design, tracks guarantee a higher mobility in difficult terrain. A combination of a snakelike robot with tracks therefore seems to be the most promising solution, given some changes compared to the previous designs.

The main problem in the most advanced previous system [7] is the conversion from electrical energy into pressure for pneumatics. For this reason the main focus in our work consisted in developing a joint with an innovative actuation principle which only needs electrical power, but is almost as lightweight as the pneumatic solution.

\section{Basic concept}

To sum up, the goal of this project was set to realize an innovative snakerobot with tracks on all sides of its segments (similar to [7]), with an actuation mechanism for the joints which does not need pneumatic supply; and to realize this robot in a robust and lightweight design and prove its functionality in realistic test-environments.

The basic mechanical concept of the robot can be seen in Figure 2. It consists of 5 similar, $30 \mathrm{~cm}$ long elements with motorized tracks on each side; 4 joints with 2 DOF each in between, a "head-unit" with camera and the cable which connects it to the control unit. For controlling it, we use angles given by a remote control interface. The electronics implemented in the system control the motors in the defined positions. 


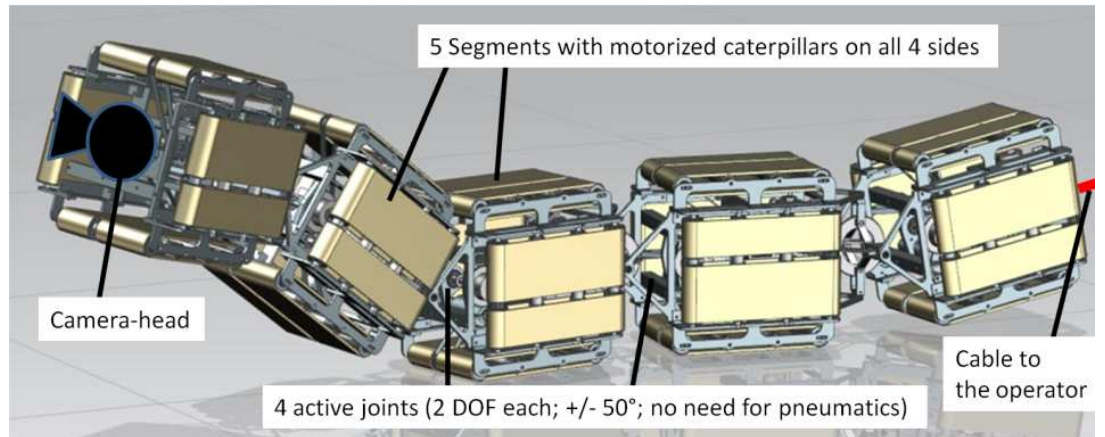

Figure 2. Overall mechanical design with the main assemblies.

\section{Detailed design}

Within the detailed design, the following challenges had to be solved: Realizing a direct-driven joint-mechanism with 2 DOF and at least $+/-45^{\circ}$, driving the tracks in each segment with only one motor and a robust and lightweight torque-transmission, controlling all motors including the camerahead, and providing an intuitive user-interface to the operator.

\subsection{Joints}

An important part of the system is the joint mechanism that connects the elements of the snakelike system. According to the goals that Traloc should climb over steps and gaps in the range of $0.5 \mathrm{~m}$, the joint has to carry high loads. After studying several load cases, the expected turning moment on the joint was specified as $21 \mathrm{Nm}$ and the sheer force as $120 \mathrm{~N}$; while the maximum total mass for each joint with $+/-45^{\circ}$ minimum-angle in 2 directions was defined to be less than $1.5 \mathrm{~kg}$ (40\% of the total weight per element).

We have considered various concepts to fulfill these specifications. Nevertheless, no satisfying solutions based on pneumatic or hydraulic actuation could be found. The reasons are that commercially available compressors with enough pressure would be too big for fitting on the robot; and externally generating the pressure and feeding it to the robot via a cable (as implemented in the Omni-Tread [7]) was regarded as unsuitable for this application. To solve this problem, we developed an innovative mechanism based on the principle of a cardan-joint that is able to generate high forces and reaches a bending angle of up to $50^{\circ}$ between two elements. The two degrees of freedom are mechanically decoupled and each of them is steered independently. All elements include two similar units with a gear-motor (Maxon EC-max25 with GP 32C 1:190, 6Nm) and a secondary transmission with two chain-drives (1:15), resulting in $42 \mathrm{Nm}$ of maximum torque on the joint and a turning-time of approximately 2.5 seconds for $45^{\circ}$. 


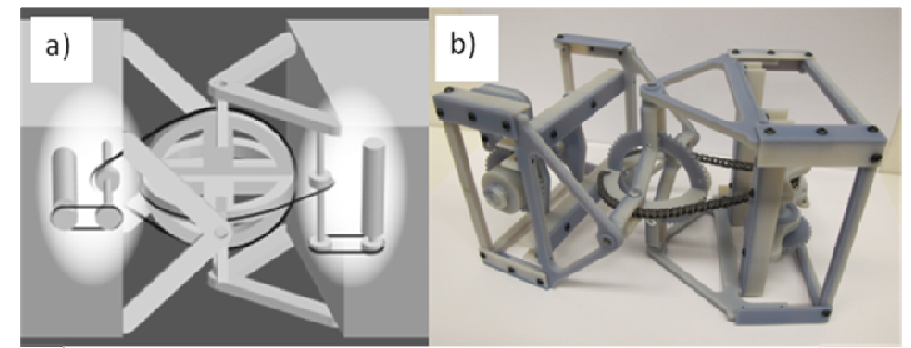

c)

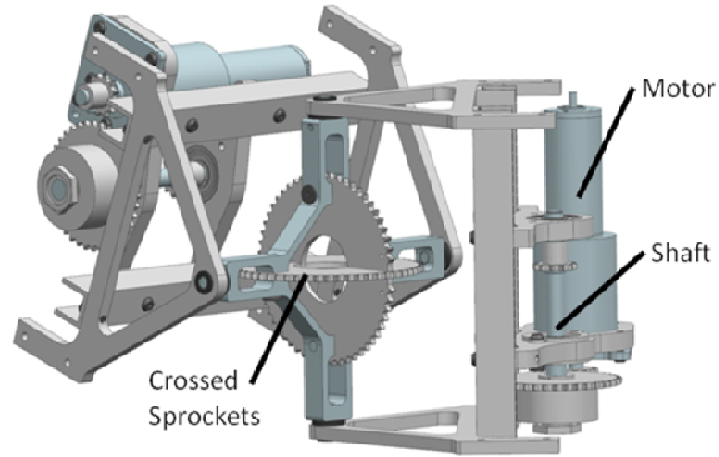

Figure 3. Joint actuation principle with direct chain-drive:

(a) Conceptual drawing of the actuation-principle, (b) Picture of a preliminary plastic model (c) Detailed Cut-view showing the main components (chains not displayed).

\subsection{Drive-train}

The drive train is essential to move the system with a reasonable number of elements. The decision to place tracks on all 4 sides of the element has been taken to increase the propulsion rate (= powered surface / total surface) and to assert the capability of driving on each side. Moreover the probability of getting stuck decreases by increasing the powered surface.

All tracks of one element are driven by one motor placed at the center of it, similar as in [7]. To transmit the power from the motor to the tracks we developed a distribution gearbox which consists of two stages combining a worm and a belt gear. As there are four tracks, the worm transfers the torque to four worm wheels which are connected to toothed belt wheels. With these four belt wheels, the connection between the drive at the center and the outer tracks is established. The distribution gear has an overall gear transmission ratio of 1:44. As Motor we chose the EC 32 flat with integrated electronics from Maxon Motor. It has a maximal rotational speed of $6000 \mathrm{rpm}$ and nominal torque of $34 \mathrm{mNm}$. Put in relation with the overall gear transmission ratio this results in a maximal moment of about $1.5 \mathrm{Nm}$ and $140 \mathrm{rpm}$ for the tracks. The apparent asymmetry of the tracks results from the internal construction of the gear. The tracks consist of four axes whereof two can be flexibly positioned to enable the 
pre-stressing of the tracks. The other two are fixed in the middle of the drive train to ensure that the timing belt won't be bended too much when driving over an edge. The tracks are manufactured of double-side polyurethane timing belts in trapezoidal shape.

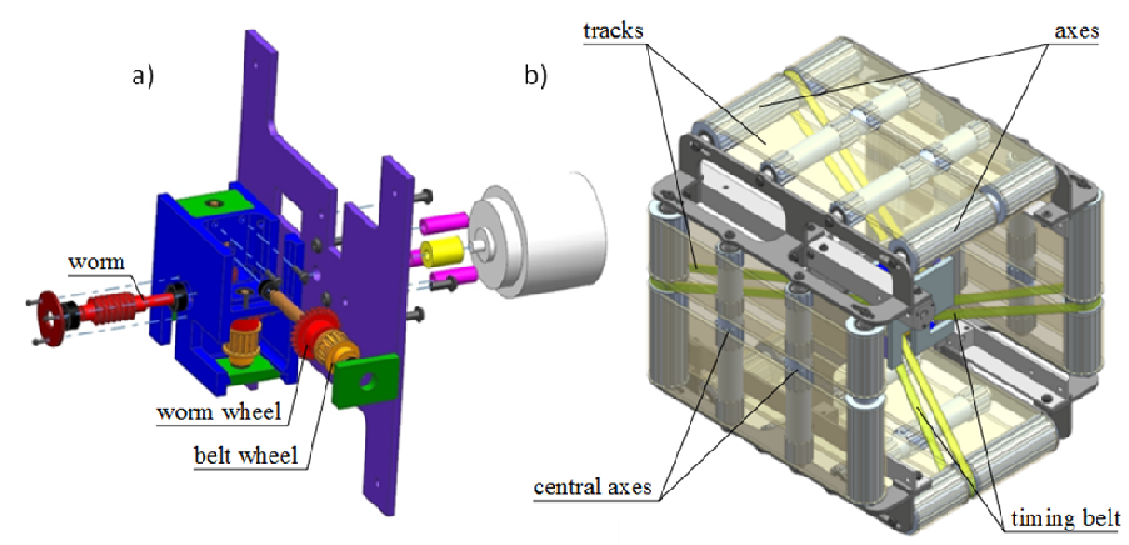

Figure 4. Drive-train for axial motion, (a) detail view of distribution gear,

(b) overview with all four tracks.

\subsection{Control and user-interface}

With respect to the electronics, each element is built the same way: There are two brushless DC-motors to drive the joints and a third one to power the train-drive. Furthermore, a magnetic rotary encoder in each joint measures the current angle. With this information, a processor controls the angle in the two joints as well as the drive speed.

This concept gives the freedom to treat each element separately and to operate the snake with a different number of elements. If elements are put together, the control units in each element can communicate with one another and exchange information. Then, one of the elements operates as master and can be connected to a remote controller from where desired reference values for the joint angles can be received and passed to all other elements.

For additional control, an IMU equipped with a gyroscope, a compass and acceleration sensors is placed in one element in order to get information about the current orientation.

Two approaches are realized for the steering of the snake. The first one is based on a small model of the snake with encoders in each joint. The robot follows the movements the person does with the model. The second variant is to drive trajectories in the plane between several given points. 


\section{Tests}

The first test with two elements proved that the concept of the joints and the drive train is working. The robot was able to drive curves as well as to lift one element. Further tests showed that Traloc is able to overcome a step of $40 \mathrm{~cm}$ and a gap of $60 \mathrm{~cm}$. Also the functionality of the steering with the model and the movable stereovision camera in the head has been demonstrated.
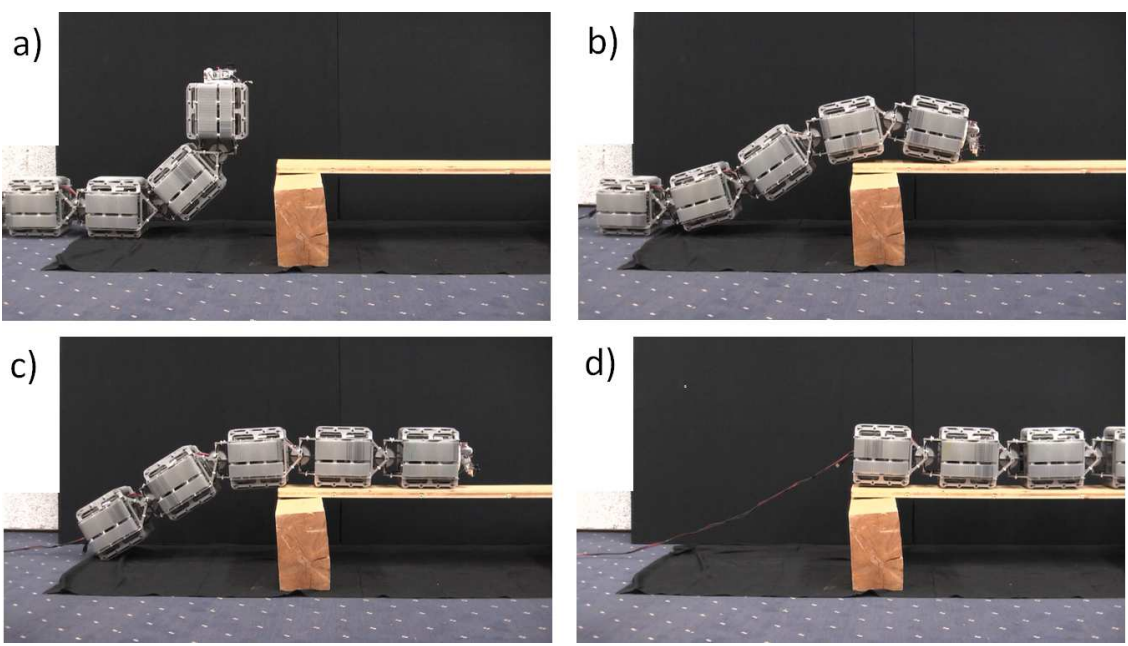

Figure 5. Sequence of the robot passing a step.

\section{Conclusion and Outlook}

The snake-robot with tracks on its elements, which was presented in this paper, illustrates an innovative prototype-design for a field-robot with high mobility in search-and-rescue-applications. Thanks to its directly-driven joints, its lightweight drive for the tracks and its intuitive control, it achieves outstanding performance and should become an important, additional tool in future missions for searching victims in collapsed buildings.

Future improvements in a final industrial version will mainly stress on the resistance against dust and water, the energy supply with batteries or a cable reel on the robot itself and the controllability of the system. 


\section{Acknowledgement}

We want to thank the technical support-team at ETH Zurich (Marco Steiger, Martin Schütz, Jean-Luc Emery: CAD-FEM; Markus Bühler, Dario Fenner: Mechanical workshop; Stefan Bertschi, Thomas Baumgartner: Electronics; Roland Haas: coordination and coaching). Further on we thank our sponsors in particular Maxon Motor, Qualicut, LW-Basel, ABB, Ruag and Armasuisse.

\section{References}

1. D. Schafroth, S. Bouabdallah, C. Bermes, R. Siegwart, "From the Test Benches to the First Prototype of the muFly Micro Helicopter", Journal of Intelligent and Robotic Systems, 2008.

2. M. Lauria, Y. Piguet, R. Siegwart, "Octopus - An Autonomous Wheeled Climbing Robot", Proc. of The Fifth International Conference on Climbing and Walking Robots (CLAWAR), 2002.

3. A. Masayuki, T. Takayama, S. Hirose, "Development of 'Souryu-III': Connected Crawler Vehicle for Inspection inside Narrow and Winding Spaces", Proceedings of 2004 IEEORSJ International Conference on Intelligent Robots and Systems, September 28 - October 2, Sendai, Japan, p. 52-57, 2004

4. C. Wright, A Johnson, A. Peck, Z. McCord, A. Naaktgeboren, P. Gianfortoni, M. Gonzalez-Rivero, R. Hatton, H. Choset, "Design of a Modular Snake Robot", IEEE RSJ International Conference on Intelligent Robots and Systems, October 29 - November 2, San Diego, USA, p. 2609-2614

5. K.L.Paap T.Christaller F.Kirchner, "A Robot Snake to Inspect Broken Buildings", Proceedings of the 2000 IEEE/RSJ International Conference on lntelligent Robots and Systems, p. 2079-2082

6. T. Kamegawa, T. Yamas, H. Igarashit, F. Matsuno. "Development of The Snake-like Rescue Robot 'KOHGA'." Proceedings of the 2004 IEEE International Conference on Robotics 8 Automation, New Orleans, LA April 2004, p. 5081-5086

7. G. Granosik, M. Hansen, J., "The OmniTread Serpentine Robot for Industrial Inspection and Surveillance", Industrial Robot: An International Journal, Band 32, p. 139-148, 2005. 